\title{
ESTUDO EPIDEMIOLÓGICO DOS TRAUMAS RAQUIMEDULARES POR PROJÉTIL DE ARMA DE FOGO NO HOSPITAL GERAL DO ESTADO DA BAHIA
}

\author{
EPIDEMIOLOGICAL STUDY OF TRAUMA OF SPINAL CORD TRAUMA BY A PROJECTILE \\ OF FIREARM IN THE HOSPITAL GERAL DO ESTADO DA BAHIA \\ ESTUDIO EPIDEMIOLÓGICO DEL TRAUMA DE LA MÉDULA ESPINAL POR PROYECTIL \\ DE ARMA DE FUEGO EN EL HOSPITAL GERAL DO ESTADO DA BAHIA
}

\author{
Maurício Guimaräes Pimentel ${ }^{1}$, Eduardo Gil França Gomes², Maurício Santos Gusmäo², Djalma Castro de Amorim Junior², \\ Marcus Thadeu Venâncio Simöes², Joilda Fontes Gomes², Jayme Batista Freire ${ }^{3}$, Joseph Azulay², Adauto José Santos Pedreira², \\ Orlando Espinheira Freire de Carvalho Filho ${ }^{4}$, Sérgio Murilo Andrade², Rony Brito Fernandes²
}

\section{RESUMO}

Objetivo: Realizar um levantamento de dados epidemiológicos em prontuários, avaliando a evolução neurológica dos pacientes vítimas de lesões por arma de fogo na coluna vertebral durante a internação hospitalar, que foram operados entre 1991 e 2010. Métodos: Os dados foram tabulados por meio de estatística descritiva, buscando significância estatística entre a melhora neurológica e o procedimento cirúrgico individualizado para cada caso. Dos 290 pacientes operados, 115 deram entrada no HGE com quadro clínico de paralisia sensitiva e motora, correspondendo a 39,66\% do total, sendo os pacientes Frankel A os mais prevalentes da nossa casuística. Resultados: Esses pacientes evoluíram com menor possibilidade de reversão dos déficits neurológicos, com $94,78 \%$ não apresentando melhora na alta hospitalar. Os portadores de lesão medular incompleta apresentaram evolução neurológica em 94,59 \% dos operados. Conclusão: Não foi encontrada significância estatística para a meIhora neurológica com relação aos procedimentos cirúrgicos realizados nos pacientes vítimas de lesões por arma de fogo na coluna vertebral.

Descritores: Lesão medular; Ferimentos por arma de fogo; Epidemiologia.

\begin{abstract}
Objective: Conduct a survey of epidemiological data in medical records, assessing the neurological outcome of patients who suffered gunshot injuries in the spine during hospitalization and who underwent surgery between 1991 and 2010. Methods: The data were tabulated through descriptive statistics, looking for statistical significance between the neurological improvement and the surgical procedures for each individual case. Of the 290 patients operated on, 115 were admitted to the HGE with clinical sensory and motor paralysis, representing 39.66\% of the total, being patients Frankel A the most prevalent in our series. Results: These patients had less possibility of reversal of neurological deficits, with $94.78 \%$ showing no improvement at discharge. Patients with incomplete spinal cord injury presented neurological outcome in $94.59 \%$ of those operated on. Conclusion: There was no statistical significance for the neurological outcome in relation to the surgical procedures performed on patients suffering gunshot injuries in the spine.
\end{abstract}

Keywords: Spinal injuries; Wounds, gunshot; Epidemiology.

\section{RESUMEN}

Objetivo: Realizar un estudio de datos epidemiológicos en las historias clínicas, evaluando la evolución neurológica de los pacientes que han sufrido lesiones de proyectil de un arma de fuego en la columna vertebral durante la hospitalización, que fueron sometidos a cirugía entre 1991 y 2010. Métodos: Se tabularon los datos a través de estadística descriptiva, en busca de significación estadística entre la mejoría neurológica y el procedimiento quirúrgico para cada caso individual. De los 290 pacientes operados, 115 ingresaron en el HGE con cuadro clínico de parálisis sensorial y motor, que representan el 39,66\% del total, siendo los pacientes Frankel A los más prevalentes de nuestra serie. Resultados: Estos pacientes evolucionaron con menos posibilidad de reversión del déficit neurológico, y el 94,78\% no mostró mejoría cuando dados de alta. Los pacientes con lesión medular incompleta mostraron resultados neurológicos en 94,59\% de los operados. Conclusiones: No se encontró significación estadística de la mejoría neurológica en respeto a los procedimientos quirúrgicos realizados en pacientes que sufren de heridas de bala en la columna vertebral.

Descriptores: Médula espinal; Heridas por arma de fuego, Epidemiología.

\section{INTRODUÇÃO}

O aumento da violência entre a sociedade civil é tema constante em todos os meios de comunicação, sendo crescente o número de pacientes que chegam às emergências vítimas de lesões por projétil de arma de fogo (PAF) na coluna vertebral. Estima-se que a cada ano, cerca de $17 \%$ dos traumas raquimedulares sejam causados por tal mecanismo ${ }^{1,2}$, correspondendo a segunda causa mais comum de lesões medulares, superado apenas pelos acidentes automobilísticos $^{3}$. As lesões por PAF podem acometer qualquer segmento vertebral, sendo o mais comum os torácicos ${ }^{2,4}$, predominantemente em pacientes do sexo masculino entre 15 e 34 anos $^{2,4,5}$.

As lesões por projétil de arma de fogo na coluna vertebral têm

1. Médico Residente em Cirurgia da Coluna Vertebral do Hospital Geral do Estado e Hospital da Santa Casa de Misericórdia da Bahia - Salvador, BA, Brasil.

2. Ortopedista Especialista em Cirurgia da Coluna Vertebral; Médico assistente do Serviço do TRM do Hospital Geral do Estado - Salvador, BA, Brasil.

3. Ortopedista Especialista em Cirurgia da Coluna Vertebral; Chefe do Serviço doTRM do Hospital Geral do Estado - Salvador, BA, Brasil.

4. Médico Neurocirurgião do Serviço do TRM do Hospital Geral do Estado - Salvador, BA, Brasil.

Trabalho realizado no Grupo de TRM do Hospital Geral do Estado, Salvador, Bahia.

Correspondência: Av. Princesa Isabel n 113 Apart. 1104 Bairro Barra - Salvador, BA, Brasil. 40130-030. maupimentel03@ hotmail.com

Recebido em 26/03/2012, aceito em 23/08/2012 
grande potencial de evoluir com sequelas irreversíveis ao paciente, na grande maioria em idade economicamente ativa, trazendo um importante impacto econômico para sociedade e altos custos de tratamento e reabilitação para o país s-7 $^{5-}$

Nos EUA vivem hoje uma média de 200 mil pessoas com lesões medulares, tendo as estimativas de gastos com esses pacientes uma relação direta com a gravidade da lesão e idade na época da lesão. Para exemplificar, um paciente com quadriplegia alta aos vinte e cinco anos, tem um custo médio de US\$1.350.000 aos cofres públicos. Com base nessas informações, torna-se mandatório o melhor entendimento dessas lesões devido a sua gravidade e os impactos socioeconômicos associados ${ }^{4}$.

O objetivo desse estudo é realizar um levantamento de dados epidemiológicos em prontuários avaliando a evolução neurológica durante o internamento dos pacientes vítimas de lesões por arma de fogo na coluna vertebral, operados no Serviço de Trauma Raquimedular do Hospital Geral do Estado, em Salvador-Bahia, Brasil.

\section{MATERIAL E MÉTODOS}

Foi realizado estudo retrospectivo de revisão de prontuário e analisados dados clínicos e epidemiológicos dos pacientes atendidos no Hospital Geral do Estado da Bahia (HGE), no período de 1991 a 2010. O Serviço de Trauma Raquimedular (TRM) do HGE é o centro de referência de todo o Estado da Bahia, onde são atendidos pacientes com lesões na coluna vertebral vindos diretamente da unidade de emergência ou regulados das demais cidades do interior do Estado.

Todos os pacientes com suspeita de lesão por PAF na coluna vertebral foram criteriosamente examinados e submetidos à radiografia simples em incidências ortogonais, associado ao exame de tomografia computadorizada do segmento vertebral afetado, com cortes axiais, coronais e reconstrução tridimensional.

$\mathrm{Na}$ grande maioria dos casos, os exames de imagem descritos, juntamente com o exame neurológico criterioso, foram suficientes para definir diagnóstico e conduta quanto a tratamento conservador ou cirúrgico. Apenas em alguns casos individualizados, tornou-se necessário o exame de ressonância nuclear magnética (RNM) do segmento para melhor definição de conduta.
A escala de Frankel foi utilizada para avaliação de déficit neurológico, de acordo com nível de lesão, na admissão e alta hospitalar dos pacientes operados vítimas de lesão por PAF na coluna vertebral.

Os dados coletados dos prontuários foram tabulados por meio de estatística descritiva utilizando variáveis discretas, buscando significância estatística a partir da análise individualizada de cada variável e um cruzamento das suas associações. Utilizamos o teste $t$ de Student sendo considerados valores de $p<0,05$ como estatisticamente significativos.

O protocolo de tratamento instituído no Serviço de TRM do HGE segue o preconizado por Barros Filho et al. ${ }^{4}$, sendo o tratamento cirúrgico empregado nos ferimentos com orifício de entrada posterior e fratura da lâmina associada, que provocaram compressão medular extrínseca, ou nos casos de PAF intramedular. Em ambos os casos, realiza-se à laminectomia associada à retirada dos fragmentos ósseos e do projétil. A artrodese é considerada de acordo com os critérios de instabilidade das fraturas. Waters et al. ${ }^{8}$ relatam benefícios evidentes em descompressões medulares de T12 a L4 quando comparadas ao tratamento não cirúrgico.

Os pacientes que apresentam o PAF alojado no disco intervertebral também foram abordados cirurgicamente para a retirada do projétil, uma vez que a intoxicação tardia pelo chumbo que se desprende do projétil pode ocorrer nesses casos. Os ferimentos transfixantes, sem instabilidade vertebral, foram tratados conservadoramente.

O tempo de internamento dos pacientes foi bastante variável de acordo com a presença ou não de complicações clínicas pré e pós-operatórias, sendo as mais prevalentes a infecção urinária, infecção respiratória, trombose venosa profunda e escara de decúbito.

\section{RESULTADOS}

Durante o período de 1991 a 2010, foram operados duzentos e noventa pacientes vítimas de lesões por arma de fogo em diversos segmentos da coluna vertebral, no Hospital Geral do Estado da Bahia.

$\mathrm{Na}$ Tabela 1 constam os dados coletados nos prontuários dos pacientes operados durante o período supracitado, não tendo registro de piora neurológica em pós-operatório. Os pacientes com indicação de tratamento conservador foram excluídos do estudo.

Tabela 1. Dados estatísticos coletados dos prontuários dos pacientes operados de 1991 - 2010 no HGE.

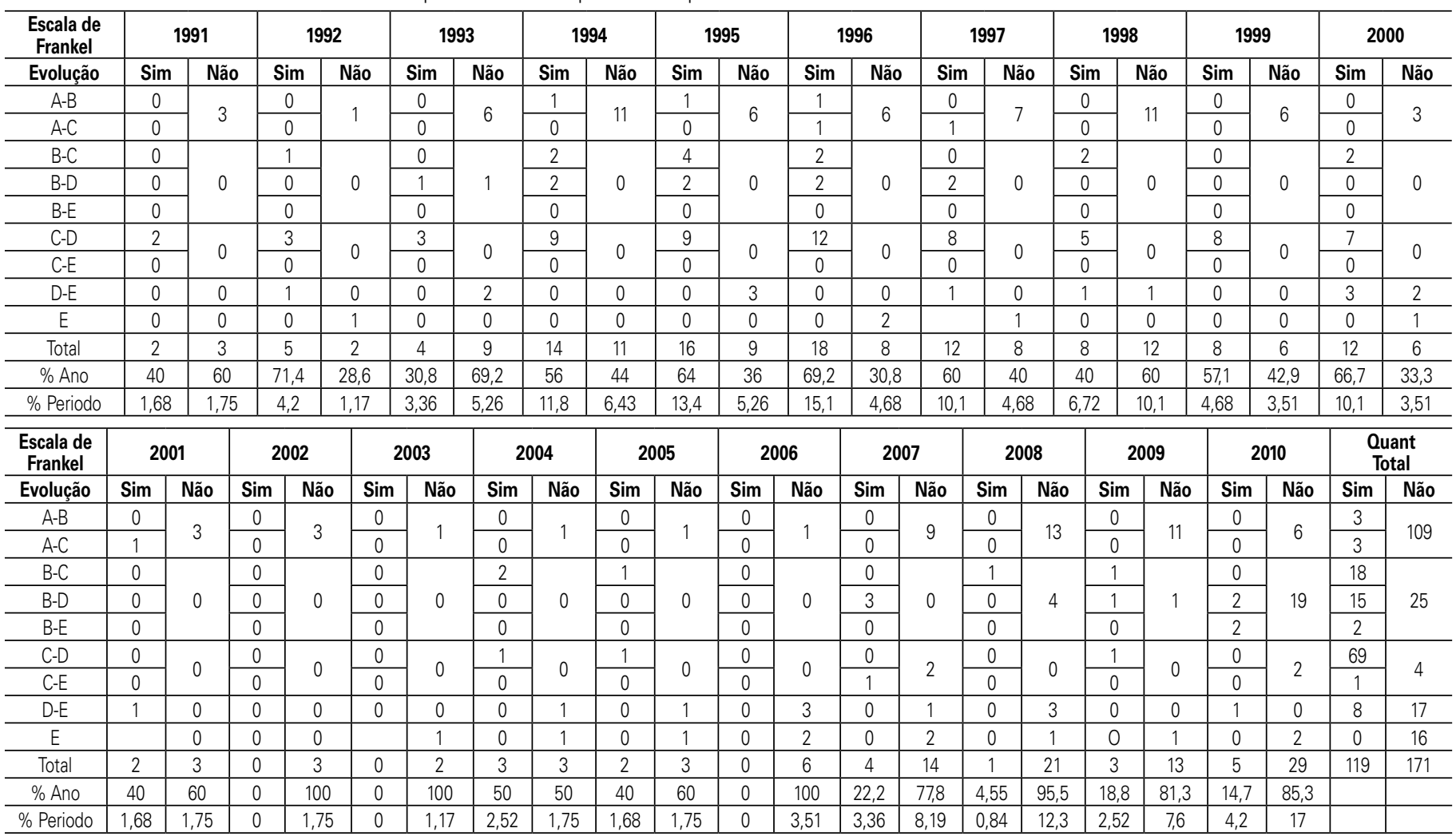


Os dados foram separados a partir da presença ou não de melhora neurológica dos pacientes operados. Na Tabela 2 constam os pacientes que mantiveram o quadro neurológica e a Tabela 3 aqueles que apresentaram alguma evolução.

De acordo com os dados das Tabelas 1,2 e 3, dos cento e quinze pacientes admitidos com lesão neurológica completa, Frankel A, observou-se que cento e nove mantiveram o nível completo de lesão no momento da alta. Apenas três pacientes evoluíram com retorno da sensibilidade, nível B da Escala de Frankel, e três obtiveram retorno de contração muscular ineficiente, Frankel C.

Sessenta pacientes foram operados com sensibilidade preservada, Frankel B. Desses, vinte e cinco não evoluíram, permanecendo com o mesmo déficit neurológico. Dezoito tornaram-se Frankel C e quinze evoluíram com retorno de contração efetiva, vencendo a força gravitacional, Frankel D. Apenas dois pacientes tiveram retorno completo da função neurológica, Frankel E.

Dos setenta e quatro pacientes Frankel $\mathrm{C}$ submetidos à cirurgia, quatro não obtiveram melhora neurológica. Sessenta e nove evoluíram com melhora parcial da força muscular, passando para Frankel $\mathrm{D}$ e apenas um paciente teve retorno completo de força muscular e sensibilidade, evoluindo para Frankel E.

Os pacientes Frankel D operados totalizaram vinte e cinco, sendo que dezessete não apresentaram evolução neurológica e oito normalizaram suas funções, evoluindo para Frankel E. Todos os pacientes operados sem déficit neurológico permaneceram no mesmo nível.

Os dados coletados foram tabulados para análise estatística, comparando a amostra que apresentou melhora neurológica (variável 1) com aquela sem evolução após as cirurgias (variável 2). Foi utilizado o teste $t$ de Student como demonstrado na Tabela 4:

$O$ resultado encontrado foi $\mathrm{P}(\mathrm{T}<=\mathrm{t})$ bi-caudal 0,198642783), um valor acima de 0,05. A partir da análise, podemos concluir que não existem grandes diferenças entres os dados fornecidos, ou seja, a diferença encontrada não aponta grande significância entre as amostras. Logo, não existe significância estatística para a melhora neurológica em função dos procedimentos cirúrgicos realizados nos pacientes vítimas de PAF na coluna vertebral.
Tabela 4. Teste t: duas amostras presumindo variâncias diferentes.

\begin{tabular}{c|c|c}
\hline & Variável 1 & Variável 2 \\
\hline Média & 5,95 & 8,55 \\
\hline Variância & 31,41842105 & 47,41842 \\
\hline Observações & 20 & 20 \\
\hline Hipótese da diferença de média & 0 & \\
\hline $\mathrm{Gl}$ & 36 & \\
\hline Stat t & $-1,309554978$ & \\
\hline $\mathrm{P}(\mathrm{T}<=\mathrm{t})$ uni-caudal & 0,099321391 & \\
\hline $\mathrm{t}$ crítico uni-caudal & 1,688297694 & \\
\hline $\mathrm{P}(\mathrm{T}<=\mathrm{t})$ bi-caudal & 0,198642783 & \\
\hline $\mathrm{t}$ crítico bi-caudal & 2,028093987 & \\
\hline
\end{tabular}

\section{DISCUSSÃO}

Este estudo avaliou duzentos e noventa pacientes vítimas de lesão por arma de fogo na coluna vertebral que foram submetidos a procedimentos cirúrgicos individualizados de acordo com a personalidade das lesões, no Hospital Geral do Estado entre 1991 e 2010.

Os pacientes foram examinados, de acordo a escala de Frankel em sua admissão e alta hospitalar ao longo de vinte anos (Figura 1).

Em virtude dos vários mecanismos que determinam as lesões no paciente atingido por um projétil de arma de fogo, as lesões que atingem a vértebra geralmente comprometem a medula espinhal e/ ou as raízes nervosas, sendo muito raro um paciente com TRM por PAF não apresentar déficit neurológico parcial ou total, geralmente definitivo.

De acordo com a Figura 1, dos duzentos e noventa pacientes operados, cento e quinze deram entrada no HGE com quadro clínico de paralisia sensitiva e motora, correspondendo a $39,66 \%$ do total, sendo os pacientes Frankel A os mais prevalentes da nossa casuística. Barros Filho et al. ${ }^{4}$ em seu estudo epidemiológico sobre o assunto encontrou uma prevalência de $92,4 \%$ de pacientes com lesão completa. Já Waters et al. ${ }^{3}$ estudando 135 pacientes, encontraram $57 \%$ de pacientes portadores de lesão medular completa.

Tabela 2. Dados estatísticos dos pacientes sem melhora neurológica após a cirurgia

\begin{tabular}{|c|c|c|c|c|c|c|c|c|c|c|c|c|c|c|c|c|c|c|c|c|c|c|}
\hline \multicolumn{23}{|c|}{ Pacientes com evolução } \\
\hline $\begin{array}{l}\text { Escala de } \\
\text { FRANKEL }\end{array}$ & 1991 & 1992 & 1993 & 1994 & 1995 & 1996 & 1997 & 1998 & 1999 & 2000 & 2001 & 2002 & 2003 & 2004 & 2005 & 2006 & 2007 & 2008 & 2009 & 2010 & $\begin{array}{c}\text { Quant } \\
\text { Total }\end{array}$ & Média \\
\hline$A-B$ & 0 & 0 & 0 & 1 & 1 & 1 & 0 & 0 & 0 & 0 & 0 & 0 & 0 & 0 & 0 & 0 & 0 & 0 & 0 & 0 & 3 & 0,15 \\
\hline$B-C$ & 0 & 1 & 0 & 2 & 4 & 2 & 0 & 2 & 0 & 2 & 0 & 0 & 0 & 2 & 1 & 0 & 0 & 1 & 1 & 0 & 18 & 0,9 \\
\hline B-D & 0 & 0 & 1 & 2 & 2 & 2 & 2 & 0 & 0 & 0 & 0 & 0 & 0 & 0 & 0 & 0 & 3 & 0 & 1 & 2 & 15 & 0,75 \\
\hline C-E & 0 & 0 & 0 & 0 & 0 & 0 & 0 & 0 & 0 & 0 & 0 & 0 & 0 & 0 & 0 & 0 & 1 & 0 & 0 & 0 & 1 & 0,05 \\
\hline D-E & 0 & 1 & 0 & 0 & 0 & 0 & 1 & 1 & 0 & 3 & 1 & 0 & 0 & 0 & 0 & 0 & 0 & 0 & 0 & 1 & 8 & 0,4 \\
\hline Total & 2 & 5 & 4 & 14 & 16 & 18 & 12 & 8 & 8 & 12 & 2 & 0 & 0 & 3 & 2 & 0 & 4 & 1 & 3 & 5 & 119 & \\
\hline
\end{tabular}

Tabela 3. Dados estatísticos dos pacientes com melhora neurológica

\begin{tabular}{|c|c|c|c|c|c|c|c|c|c|c|c|c|c|c|c|c|c|c|c|c|c|c|}
\hline \multicolumn{23}{|c|}{ Pacientes sem evolução } \\
\hline $\begin{array}{l}\text { Escala de } \\
\text { FRANKEL }\end{array}$ & 1991 & 1992 & 1993 & 1994 & 1995 & 1996 & 1997 & 1998 & 1999 & 2000 & 2001 & 2002 & 2003 & 2004 & 2005 & 2006 & 2007 & 2008 & 2009 & 2010 & $\begin{array}{l}\text { Quant } \\
\text { Total }\end{array}$ & Média \\
\hline$A-A$ & 3 & 1 & 6 & 11 & 6 & 6 & 7 & 11 & 6 & 3 & 3 & 3 & 1 & 1 & 1 & 1 & 9 & 13 & 11 & 6 & 109 & 5,45 \\
\hline $\mathrm{C}-\mathrm{C}$ & 0 & 0 & 0 & 0 & 0 & 0 & 0 & 0 & 0 & 0 & 0 & 0 & 0 & 0 & 0 & 0 & 2 & 0 & 0 & 2 & 4 & 0,20 \\
\hline D-D & 0 & 0 & 2 & 0 & 3 & 0 & 0 & 1 & 0 & 2 & 0 & 0 & 0 & 1 & 1 & 3 & 1 & 3 & 0 & 0 & 17 & 0,85 \\
\hline
\end{tabular}


Quanto ao prognóstico neurológico das vítimas de PAF em coluna vertebral, os pacientes com lesão completa evoluíram com menor possibilidade de reversão dos déficits neurológicos, com $94,78 \%$ não apresentando evolução na alta hospitalar. Apenas $5,22 \%$ desses pacientes apresentaram melhora, entretanto não atingindo capacidade deambulatória como demonstrados na Tabela 5 e Figura 2.

Dos sessenta pacientes Frankel B submetidos à cirurgia, 58,33 \% obtiveram evolução neurológica favorável. Já os pacientes Frankel C, com total de setenta e quatro, observou-se evolução neurológica em $94,59 \%$ dos pacientes operados. Houve melhora em $32 \%$ dos pacientes Frankel D submetido a cirurgias. Não houve piora neurológica nos pacientes operados sem déficit neurológico.

De acordo com a Figura 2, os pacientes com lesões neurológicas incompletas foram os mais beneficiados com os procedimentos cirúrgicos obtendo melhora neurológica mais significativa, principalmente os admitidos com Frankel C.

Em seu estudo epidemiológico sobre o assunto, Barros Filho et al. ${ }^{4}$ encontraram um percentual pequeno de pacientes com déficit neurológico incompleto. Dos 3,7 \% referido no seu estudo, a maioria tinha seus ferimentos em coluna lombossacra com lesão parcial e assimétrica da cauda eqüina e 3,9\% não apresentavam déficit. Waters et al. ${ }^{3}$ relatam uma prevalência de $47 \%$ de pacientes com déficits parciais. Entretanto, não há referência em relação à recuperação neurológica em nenhum dos dois estudos supracitados.

A despeito da prevalência de pacientes portadores de lesão medular completa ser maior, tanto no nosso estudo como no dos outros autores supracitados, é importante salientar a grande discrepância na percentagem de pacientes portadores de lesão medular completa e lesão incompleta, quando se compara nossa casuística com a dos dois autores.

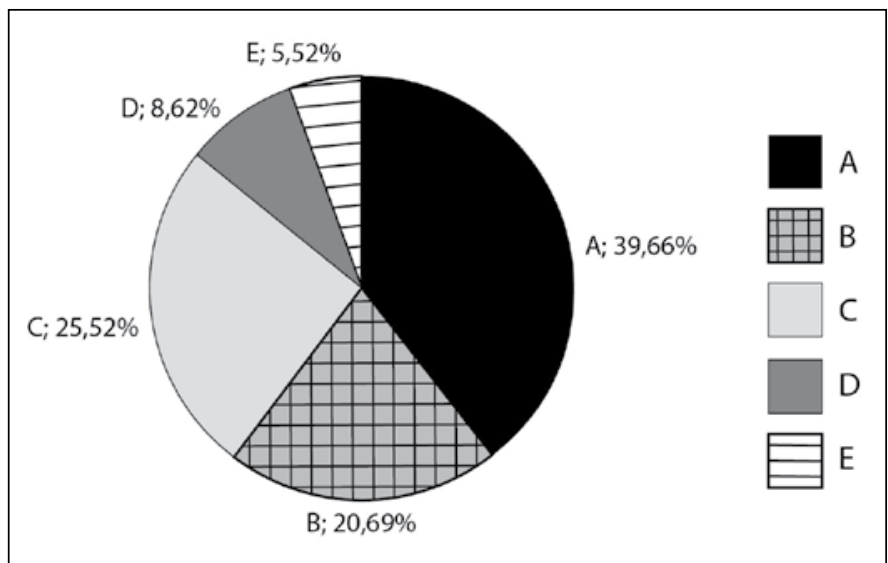

Figura 1. Percentual de pacientes na admissão segundo escala de Frankel.

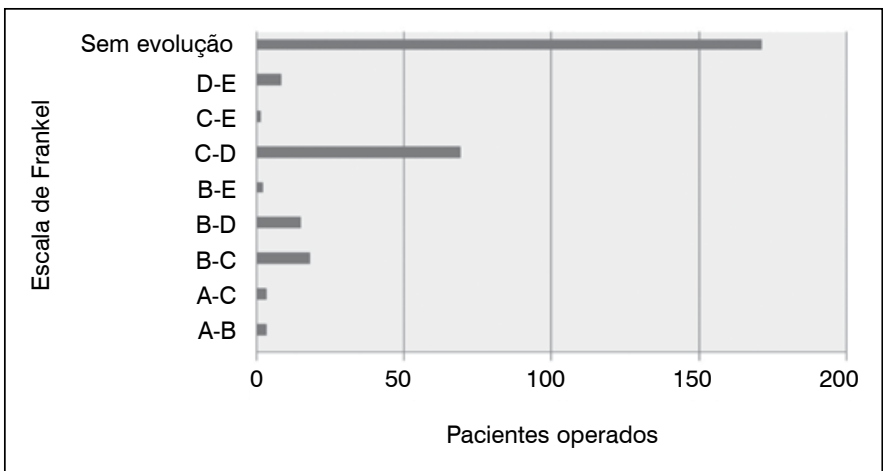

Figura 2. Melhora neurológica dos pacientes operados segundo escala de Frankel.

Tabela 5. Percentual de evolução neurológica dos pacientes operado separados por subgrupo de acordo com a escala de Frankel da admissão.

\begin{tabular}{|c|c|c|c|c|c|c|c|c|c|c|c|}
\hline & -rankel & & & Frankel & & & Frankel & & & Frankel L & \\
\hline Dentr & $\begin{array}{l}\text { ro do } m \\
\text { grupo }\end{array}$ & nesmo & Dent & $\begin{array}{l}\text { ro do } m \\
\text { grupo }\end{array}$ & nesmo & Dent & $\begin{array}{l}\text { ro do } m \\
\text { grupo }\end{array}$ & smo & Dentr & $\begin{array}{l}\text { ro do } m \\
\text { grupo }\end{array}$ & esmo \\
\hline Evoluiu & Quant & Percent & Evoluiu & Quant & Percent & Evoluiu & Quant & Percent & Evoluiu & Quant & Percent \\
\hline Sim & 6 & 5,22 & Sim & 35 & 58,33 & Sim & 70 & 94,59 & Sim & 8 & 32,00 \\
\hline Não & 109 & 94,78 & Não & 25 & 41,67 & Não & 4 & 5,41 & Não & 17 & 68,00 \\
\hline
\end{tabular}

No Brasil a literatura científica infelizmente fornece poucas informações sobre dados epidemiológicos das fraturas vertebrais e lesões medulares, dificultando a adoção de políticas públicas de prevenção e assistência aos pacientes acometidos.

\section{CONCLUSÃO}

O aumento constante da incidência e gravidade do trauma raquimedular por projétil de arma de fogo nos últimos anos é motivo de grande preocupação social, devendo ser considerado pelos órgãos públicos como um grave problema de saúde pública. $\mathrm{Na}$ grande maioria dos casos, o TRM por PAF provoca sequelas graves de caráter irreversível em pacientes jovens e economicamente ativos, com prejuízo incalculável na qualidade de vida das suas vítimas e custos muito elevados para o governo.

Diante dos dados apresentados da nossa casuística dos últimos vinte anos, devidamente tabulados para análise estatística, os autores não encontraram significância estatística entre os procedimentos cirúrgicos e a melhora neurológica dos pacientes vítimas de TRM por PAF na coluna vertebral, o que ratifica o caráter irreversível das suas lesões na grande maioria dos casos.

\section{REFERÊNCIAS}

1. Kitchel SH. Current treatment of gunshot wounds to the spine. Clin Orthop Relat Res.2003;(408):115-9

2. Bono CM, Heary RF. Gunshot wounds to the spine. Spine J. 2004;4(2):230-40.

3. Waters RL, Sie IH. Spinal cord injuries from gunshot wounds to the spine. Clin Orthop Relat Res. 2003;(408):120-5.

4. Barros Filho TEP, Oliveira RP, Barros EK, Von Uhlendorff EF, lutaka AS, Cristante AF, et al. Ferimentos por arma de fogo na coluna vertebral: estudo epidemiológico.Coluna/ Columna. 2002;1(2):83-7.

5. National Spinal Cord Injury Statistical Center. Spinal cord injury facts and figures at a

Glance. Birmingham: University of Alabama; 1999

6. Zaninelli EM, Graells XSI, Néri OJ, Dau L. Avaliação epidemiológica das fraturas da coluna torácica e lombar de pacientes atendidos no Pronto-Socorro do Hospital do Trabalhador da UFPR de Curitiba - Paraná. Coluna/Columna. 2005;4(1):11-5.

7. Ebraheim NA, Savolaine ER, Jackson WT, AndreshakTG, Rayport M. Magnetic resonance imaging in the evaluation of a gunshot wound to the cervical spine. J Orthop Trauma. 1989;3(1):19-22.

8. Waters RL, Adkins RH, Yakura J, Sie I.Profiles of spinal cord injury and recoveryafter gunshot injury. Clin Orthop Relat Res. 1991;(267):14-21 